\title{
Effect of Activities of Daily Living Strategy for Post Stroke Patient on Degree of Spasticity at Minia University Hospital
}

\author{
Hend Elham Mohamed Zaky, Zienab Abd EL-Lateef Mohammad, Abdou Saad Taha EL-Labban and Gehan \\ Ahmed Gamal EL-Dein
}

Medical - Surgical Nursing, Faculty of Nursing, Minia University,

Medical/Surgical Nursing, Faculty of Nursing, Assiut University,

Rheumatology and Rehabilitation, Faculty of Medicine, Minia University,

Medical/Surgical Nursing, Faculty of Nursing, Minia University Abstract

\begin{abstract}
Back ground: Stroke is a leading cause of disability. Rehabilitation aims to hasten and maximize recovery from stroke by treating the disabilities caused by the stroke. The aim of this study determines the effect activities of daily living strategy for post stroke patient on degree of spasticity. Quasi-experimental research design was utilized to meet the aim of this study. The study sample included 60 adult patients with stroke they were allocated randomly into two groups, study and control. Setting Rheumatology and Rehabilitation Department at Minia University Hospital. Three tools were utilized for data collection; Pre/post test knowledge questionnaire sheet, Barthel index scale, Ashworth scale. Results of the study documented a significant improvement in knowledge and practices post strategies in study group in addition to reaching of an optimum level of independence for daily activities living and improvement degree of spasticity in study group more than control group,. In conclusion, education of stroke patient is necessary to achieve an optimum level of functioning. Replication of this study on larger probability sample is highly recommended.
\end{abstract}

\section{Key wards: Activities of Daily Living Strategies, Stroke \& Patient's out comes.}

\section{Introduction}

Stroke defined according to National Institute of Neurologic Disorder and Stroke (NINDS) and the World Health Organization (WHO) as loss of the brain function related to inadequate cerebrovasculer blood flow more than 24 hour (Carison, 2009).

The stroke is leading causes of death and disability in many developed nations. In the United States, it is the third leading cause of death, after heart disease and cancer. There are approximately 750,000 cases of stroke each year and 160,000 are fatal. Approximately 4 million people in the United States have survived a brain attack and are living. Although stroke is considered a condition of elderly, stroke can happen to any one, $28 \%$ of their victims are under the age 65years. The annual cost of ischemic stroke is approximately $\$ 71.8$ billon. The prevalence of stroke is almost twice as high for African Americans than Caucasians, and more men than women experience stroke. However, women account for approximately $60 \%$ of all stroke deaths. Stroke kills almost twice as many women as breast cancer (Osborn, et al., 2010). In Upper Egypt, determine the characteristics of hospitalized stroke patients and case fatality in Upper Egypt governorates areas (Sohag, Quena and Aswan). Abbas, et al., (2009) reported higher prevalence of hemorrhagic strokes reported in $37.5 \%$ of cases. $56 \%$ were above 60 years and $8.6 \%$ were juvenile events. $62 \%$ of stroke occurred in autumn-winter.
Hemorrhagic stroke was higher in spring-summer (53\%). Hypertension was the more frequent risk factor reported in $42 \%$ of patients.

Strokes can be classified into two major categories: ischemic and hemorrhagic. Ischemic strokes are those that are caused by interruption of the blood supply, while hemorrhagic strokes are the ones which result from rupture of a blood vessel or an abnormal vascular structure. About $85 \%$ of strokes are caused by ischemia while hemorrhagic stroke reprsents approximately $15 \%$ of all strokes (Donnan et al., 2008).

Risk factors can be divided into nonmodifiable and modifiable. Stroke risk increases with multiple risk factors. Nonmodifiable risk factors include age, gender, ethnicity/race, and family history/heredity. Stroke risk increases with age, doubling each decade after55 years of age. Two third of all strokes occur in individuals older than 65years, but stroke can occur at any age. Strokes are more common in men, but more woman die from stroke than men, because the woman tend to live longer than men; they have more opportunity to suffer a stroke (Lewis et al., 2011).

Modifiable risk factors are that can potentially be altered through lifestyle changes and medical treatment, thus reducing the risk for stroke. Modifiable risk factors include hypertension, heart disease, smoking, excessive alcohol consumption, 
obesity, sleep apnea, metabolic syndrome, and lack of physical exercises, poor diet and drug abuse (Haley, 2010).

The warning signs of stroke are any of the following conditions that occur suddenly which need Immediate medical attention; sudden weakness and numbness of the face, arm or leg most often on one side of the body, sudden dimness or loss of vision, especially in one eye, loss of speech or difficulty understanding speech, sudden sever headache without known cause, unexplained unsteadiness, dizziness, or sudden falls, especially with one of other symptoms. Therefore, each person may have different stroke warning signs (Edlin and Golanty, 2010).

Asghar et al., (2009) stated that, Stroke survivors develop their own strategies to combat disabilities, as a making an appropriate strategy is an important step in improving life for many stroke survivors and may affect the success of rehabilitation and minimize the negative effects of recurrent stroke to the stroke patient. Participants valued better knowledge and skills regarding the adaptive strategies for stroke survivors are essential in accomplishing with activities of daily living and doing social roles for improving life after stroke. Also developing the socio- economic supports is crucial for assuring a more supportive approach to achieve rehabilitation services and design better educational program for them. Therefore, the present study has carried out in an attempt to investigate the impact of implementing strategies of daily living rehabilitative activities for post stroke patients at Minia University Hospital.

Anthony, (2012) mentioned that, spasticity is a common feature of the upper motor neuron syndrome following stroke. It can have a disabling effect on the stroke survivor through pain and reduced mobility, which may limit the potential success of rehabilitation. Spasticity can also affect quality of life (QoL) and can be both diverse and highly detrimental to daily functioning. Spasticity can result in urinary incontinence; limit sexual intimacy; interfere with walking, sitting, and standing; and generally reduce a person's ability to undertake activities of daily living ADLs.

\section{Significance of the study}

Stroke is a condition with high incidence and mortality rates, leaving a large proportion of survivors with significant residual physical and psychological impairments. Through the previous of 2 years from 2008 to 2010 inpatient hospital's recorded 255 patients come to rheumatology and rehabilitation department at Minia University Hospital. The increasing numbers of older adults for acute stroke suggest that will be an increase in the number of stroke survivors living with disabilities. This increased demands on rehabilitation efforts and services, making the issue of how to best limit strokerelated disability and health risks a major concern for healthcare providers. At this time efforts to prevent stroke must be balanced with the pragmatic effort to prevent disability and maximize quality of life for those who have suffered the consequences of stroke. So, this study will be carried out to investigate the impact of implementing strategies of daily living rehabilitative activities for post stroke patients on degree of spasticity at Minia University Hospital.

\section{Subjects and Methods}

\section{Research question}

Are activities of daily living strategy effect on post stroke patient on degree of spasticity?

\section{Research design}

Quasi-experimental research design was utilized to meet the aim of the study.

\section{Technical design}

\section{Setting of the study}

This study was carried out in Rheumatology and Rehabilitation Department at Minia University Hospital.

Subjects

\section{Inclusion \& Exclusion criteria}

Sixty convenient adult stroke patients and their age ranged between 20-64 years will be selected according to the following criteria: patients with stroke who are free from sever cognitive, physical and communication impairment and study of comorbideties such as arthritis, diabetic neuropathy and musculoskeletal problem and didn't receive any program related stroke rehabilitation. The patient will be divided randomly into two equal groups (study and control).

Study Tools: Data pertinent to the study were collected, utilizing three tools:

1- Pre/post test questionnaire sheet for the patient about stroke and activities of daily living after stroke disease in additional to sociodemographic and medical data sheet for patient.

2- Barthel index to assess activities of daily living performance.

3- Ashworth score to assess degree of spasticity.

Tool I Pre/post test questionnaire sheet

Interview questionnaire sheet was constructed after reviewing the relevant literature (Rodgers and Kowalski (2008), Carlson (2009), Kluwer (2009) and Sole, et al., (2009) that was designed by the researcher to assess Patients' knowledge regard his disease. It was developed and translated into arabic language. The questionnaire sheet was administered by the researcher to the Patients' for answering all its components (pre/post tests immediately and after 2 month) the total number of questions was 39. 
Pre/post test questionnaire sheet concerning two parts

A-Sociodemographic and medical data sheet will be developed by the investigator and revised by supervisors, this covered the following:

1- Patient age, sex, occupation, education level, address and diagnosis.

2- Medical past history suffering from chronic disease such as (cardiovascular disease, diabetes mellitus and respiratory disease) and previous neurological disease such as if patients' history for cerebral infarction, cerebral hemorrhage, transient ischemic attacks, seizure and brain tumor.

B- Patients' knowledge and practices regard his disease; this part covered the following information:

1. Patients' knowledge about concept of stroke, it was included 19 questions.

2. Patients' knowledge about activities of daily living performance after stroke disease, it was included 20 questions.

\section{Scoring system}

for patients' knowledge was (39) degree, score of one was given for correct answer and a zero for incorrect answer.

Tool II: Activities of Daily Living (ADL) performance: This tool will be assessing by the Barthel index. It uses ten variables describing activities of daily living (Mobility (on level surfaces, Stairs, Transfers (bed to chair and back), Toilet use, Bladder, Bowels, Dressing, Grooming, Bathing, Feeding) were adopted from (Mahony and Barthel, 1965).

Scoring system, full credits have score (100) when patient not need minimal help or supervision during performance and physically independent, while a score of (75-90) is given when patient mildly disabled, subsequently a score of (50-70) is given when patient moderately disabled, while a score of (25-45) severely disabled and a score of (0-20) is given when patient very severely disabled and can not perform activities of daily living

Tool III: Clinical assessment of degree of spasticity according to Ashworth Score then stratification of the patient according to spasticity degree were adopted from (Ashworth, 1964).

Scoring system, no increase in muscle tone have score (0), while a score of (1), is given when slight increase in tone giving a "catch" when affected part is moved in flexion or extension, but a score of (2), is given when more marked increase in tone but affected part is easily flexed, subsequently a score of (3), is given when considerable increase in tone; passive movement difficult and a score of (4), is given when limb rigid in flexion or extension.
Educational and Training booklet was prepared by the researcher based on the knowledge and skills needs. It was also supplemented with information based on review of relevant literature (Carlson. (2009), Black and Hawks. (2009) and National Stroke Association. (2009) about stroke and activities of daily living for stroke patients' and the booklet was reviewed by supervisors.

Operational design

Methods was concerning formulation of the study tool by the researcher based upon extensive review of related literature and then content validity of the tools was checked and revised by 5 nursing and medical experts Reliability was estimated by alpha cronbach`s test for tool one and its result was $\mathrm{R}=0.82$

Pilot study

A pilot study was carried out in November-December 2011 to test the tool. A pilot study was conducted for purpose to testing clarity, completeness and practicability of the study tools of (6) patients to determine the time involvement. Also it was done to show the possibility and effectiveness of using the study tools. Necessary changes were carried out after completing the pilot study. The researcher modified some of the questions. Some details not required were omitted, either to advance or reduce the tool lengthy test. Also to show the possibility and the effectiveness of using thd e study tools regarding the policy of hospital.

\section{Technique for data collection}

A structured interview was utilized to fill out the three tools.

\section{Phase I: Preparatory phase}

A review of current and local and international related to literature in a various aspects of the problems using books, articles, periodicals and magazines was done. The proposed study settings were assessed for the number of patients suffering from stroke.

Phase II: Implementation phase

Once permission was granted to proceed with proposed study, the researcher initiated data collection.

The study tools were carried out while the patients were done physiotherapy treatment. Each patient interview lasted for 30-45 minutes by using the direct observation technique; data were collected over a period of 12 months.

At initial interview the researcher introduce her self to initiate line of communication, explain the nature \& purpose of the study, prior to answering the questions to gain their oral consent \& cooperation, and fill out the questionnaire sheet by patients individually.

Monitoring the Barthel index and Ashworth scale was carried out while the patients were on take 
physiotherapy treatment (ultraviolet rays and rang of motion exercise). Each patient was observed by the researcher (pre educational Strategies and post immediately and after two month).

The whole period for implementation of study was morning shift. The educational strategy was conducted through 6 sessions. The duration of each session ranged from 30-45 minutes. The first 2 sessions started by theoretical part about knowledge related to meaning of stroke, its signs and symptoms, types of medication use as treatment, complications and prevention the recurrent stroke.

The later 4 sessions concerning the practical part about moving of hemparesis patient and exercise, how to wear clothes after disabilities, nutrition and elimination. Before beginning any sessions usually started by a summary of what has been taught during the previous sessions and the objectives of the new topics. The sessions were conducted by the researcher in a simple Arabic language utilizing the predetermined teaching materials, Theoretical part; the teaching program was conducted through discussion, demonstration and redemonstration. Each patient was received the illustrated booklet as a hand out theoretical and practical content. An open channel of communication was achieved between the researcher and patients to assure understanding, answer any question and to verify information and practice. The effect of the educational strategies on patient's condition was reached through comparing patients achieved levels (scores or grades) before and after the application of the educational strategies (for knowledge about (stroke and activities of daily living for stroke patient) and could increase independency measured by Barthel index and assessment degree of spasticity according to Ashworth Scale testing form. compared study group with the control group.

Ethical and legal consideration

Confidentiality and anonymity of patients was assured through coding of all data. Each patient has right to withdraw from the study at any time without any rational.

\section{Results}

The aim of the present study is to

Determine the effect activities of daily living strategy for post stroke patient on degree of spasticity.

Table (1) : Frequency and percentage distribution Socio-demographic characteristic of the studies group (control group and study group).

\begin{tabular}{|c|c|c|c|c|c|c|}
\hline \multirow{2}{*}{$\begin{array}{c}\text { Socio-demographic } \\
\text { characteristic }\end{array}$} & \multicolumn{2}{|c|}{ Control (No30) } & \multicolumn{2}{|c|}{ Study (No30) } & \multirow{2}{*}{ T. test } & \multirow{2}{*}{ p. value } \\
\hline & No & $\%$ & No & $\%$ & & \\
\hline $\begin{array}{l}\text { Sex } \\
\text { Male } \\
\text { Female } \\
\end{array}$ & $\begin{array}{l}16 \\
14\end{array}$ & $\begin{array}{l}53.3 \\
46.7 \\
\end{array}$ & $\begin{array}{l}14 \\
16\end{array}$ & $\begin{array}{l}46.7 \\
53.3 \\
\end{array}$ & -0.509 & $0.613 \mathrm{Ns}$ \\
\hline $\begin{array}{l}\text { Marital status } \\
\text { Married } \\
\text { Widow }\end{array}$ & $\begin{array}{l}20 \\
10\end{array}$ & $\begin{array}{l}66.6 \\
33.3\end{array}$ & $\begin{array}{l}17 \\
13\end{array}$ & $\begin{array}{l}56.6 \\
43.3\end{array}$ & 1.000 & $0.0001 * *$ \\
\hline $\begin{array}{l}\text { Age } \\
<20 \text { years } \\
21-40 \text { years } \\
41-50 \text { years } \\
>51 \text { to } 64 \text { years }\end{array}$ & $\begin{array}{c}2 \\
4 \\
6 \\
18\end{array}$ & $\begin{array}{c}6.7 \\
13.3 \\
20.0 \\
60.0\end{array}$ & $\begin{array}{c}- \\
6 \\
6 \\
18\end{array}$ & $\begin{array}{c}- \\
20.0 \\
20.0 \\
60.0\end{array}$ & 0.222 & $0.825 \mathrm{Ns}$ \\
\hline Mean \pm SD & \multicolumn{2}{|c|}{$51.20 \pm 12.333$} & \multicolumn{2}{|c|}{$50.03 \pm 15.041$} & & \\
\hline $\begin{array}{l}\text { Educational level } \\
\text { Illiterate } \\
\text { Write and read } \\
\text { Secondary } \\
\text { Diploma }\end{array}$ & $\begin{array}{c}26 \\
4 \\
- \\
-\end{array}$ & $\begin{array}{c}86.7 \\
13.3 \\
- \\
-\end{array}$ & $\begin{array}{c}22 \\
4 \\
2 \\
2 \\
\end{array}$ & $\begin{array}{c}73.3 \\
13.3 \\
6.7 \\
6.7 \\
\end{array}$ & 1.350 & $0.182 \mathrm{Ns}$ \\
\hline $\begin{array}{l}\text { Occupation } \\
\text { Farmer } \\
\text { Worker } \\
\text { Housewife } \\
\text { Retirement } \\
\text { Employ }\end{array}$ & $\begin{array}{c}12 \\
4 \\
14 \\
- \\
-\end{array}$ & $\begin{array}{c}40.0 \\
13.3 \\
46.7 \\
- \\
-\end{array}$ & $\begin{array}{c}6 \\
4 \\
14 \\
4 \\
2\end{array}$ & $\begin{array}{c}20.0 \\
13.3 \\
46.7 \\
13.3 \\
6.7\end{array}$ & 0.0001 & $1.000 \mathrm{Ns}$ \\
\hline $\begin{array}{l}\text { Residence } \\
\text { Urban } \\
\text { City }\end{array}$ & $\begin{array}{l}18 \\
12\end{array}$ & $\begin{array}{l}60.0 \\
40.0\end{array}$ & $\begin{array}{c}22 \\
8\end{array}$ & $\begin{array}{l}73.3 \\
26.7\end{array}$ & 1.000 & $0.0001 * *$ \\
\hline
\end{tabular}


Table (2) Frequency distribution of patients' past medical history for both groups.

\begin{tabular}{|c|c|c|c|c|c|c|}
\hline \multirow{2}{*}{ Variable } & \multicolumn{2}{|c|}{ Control (No 30) } & \multicolumn{2}{|c|}{ Study (No 30) } & \multirow{2}{*}{ T. test } & \multirow{2}{*}{ p. value } \\
\hline & No & $\%$ & No & $\%$ & & \\
\hline $\begin{array}{l}\text { Previous hospitalization } \\
\text { Yes } \\
\text { No }\end{array}$ & $\begin{array}{l}18 \\
12\end{array}$ & $\begin{array}{l}60.0 \\
40.0\end{array}$ & $\begin{array}{l}20 \\
10\end{array}$ & $\begin{array}{l}66.7 \\
33.3\end{array}$ & 0.528 & $0.599 \mathrm{Ns}$ \\
\hline $\begin{array}{l}\text { History of neurological disease } \\
\text { Cerebral infarction } \\
\text { Cerebral hemorrhage } \\
\text { TIAS } \\
\text { Cerebral infarction and TIAS } \\
\text { Infarction and hemorrhage } \\
\text { Not found }\end{array}$ & $\begin{array}{c}14 \\
2 \\
- \\
4 \\
- \\
10\end{array}$ & $\begin{array}{c}46.7 \\
6.7 \\
- \\
13.3 \\
- \\
33.3\end{array}$ & $\begin{array}{c}2 \\
- \\
2 \\
2 \\
2 \\
22\end{array}$ & $\begin{array}{c}6.7 \\
- \\
6.7 \\
6.7 \\
6.7 \\
73.3\end{array}$ & -0.627 & $0.533 \mathrm{Ns}$ \\
\hline $\begin{array}{l}\text { Time of previous neurological disease } \\
<\text { one year } \\
2<5 \text { years } \\
\text { Not found } \\
\end{array}$ & $\begin{array}{c}8 \\
12 \\
10\end{array}$ & $\begin{array}{l}26.7 \\
40.0 \\
33.3\end{array}$ & $\begin{array}{c}4 \\
4 \\
22 \\
\end{array}$ & $\begin{array}{l}13.3 \\
13.3 \\
73.3\end{array}$ & -3.043 & $0.004 * *$ \\
\hline Stroke recurrence & 20 & 66.7 & 8 & 26.6 & -3.043 & $0.004 * *$ \\
\hline $\begin{array}{l}\text { Smoking } \\
\text { Cigarette } \\
\text { Water pipe } \\
\text { Negative smoker } \\
\text { Non smoker } \\
\end{array}$ & $\begin{array}{c}14 \\
6 \\
- \\
10\end{array}$ & $\begin{array}{c}46.7 \\
20.0 \\
- \\
33.3\end{array}$ & $\begin{array}{c}2 \\
6 \\
14 \\
8\end{array}$ & $\begin{array}{c}6.7 \\
20.0 \\
46.7 \\
26.6\end{array}$ & -1.255 & $0.215 \mathrm{Ns}$ \\
\hline
\end{tabular}

Table (3) Frequency distribution of medical data for both groups.

\begin{tabular}{|c|c|c|c|c|c|c|}
\hline \multirow{2}{*}{ Variable } & \multicolumn{2}{|c|}{ Control (No30) } & \multicolumn{2}{|c|}{ Study (No30) } & \multirow{2}{*}{ T. test } & \multirow{2}{*}{ P. Value } \\
\hline & No & $\%$ & No & $\%$ & & \\
\hline $\begin{array}{c}\text { Causes of current stroke } \\
\text { Cerebral infarction } \\
\text { Cerebral hemorrhage }\end{array}$ & $\begin{array}{c}24 \\
6\end{array}$ & $\begin{array}{l}80.0 \\
20.0\end{array}$ & $\begin{array}{c}24 \\
6\end{array}$ & $\begin{array}{l}80.0 \\
20.0\end{array}$ & 0.0001 & $1.000 \mathrm{Ns}$ \\
\hline $\begin{array}{l}\text { Time of current stroke } \\
<6 \text { month } \\
7 \text { month }<1 \text { year } \\
1 \text { year to } 2 \text { years }\end{array}$ & $\begin{array}{c}18 \\
8 \\
4\end{array}$ & $\begin{array}{l}60.0 \\
26.7 \\
13.3\end{array}$ & $\begin{array}{c}24 \\
6 \\
-\end{array}$ & $\begin{array}{c}80.0 \\
20.0 \\
-\end{array}$ & 2.184 & $0.033 * *$ \\
\hline $\begin{array}{l}\text { physical disabilities from stroke } \\
\text { Left side paresis } \\
\text { Right side paresis } \\
\text { Left side paralysis } \\
\text { Right side paralysis }\end{array}$ & $\begin{array}{c}8 \\
18 \\
2 \\
2\end{array}$ & $\begin{array}{c}26.7 \\
60.0 \\
6.7 \\
6.7\end{array}$ & $\begin{array}{l}12 \\
18 \\
- \\
-\end{array}$ & $\begin{array}{c}40.0 \\
60.0 \\
- \\
-\end{array}$ & 1.964 & $0.054 *$ \\
\hline $\begin{array}{l}\text { physical disabilities effect on dominant } \\
\text { hand } \\
\text { Yes } \\
\text { No }\end{array}$ & $\begin{array}{l}18 \\
12\end{array}$ & $\begin{array}{l}60.0 \\
40.0\end{array}$ & $\begin{array}{c}22 \\
8\end{array}$ & $\begin{array}{l}73.3 \\
26.7\end{array}$ & 1.088 & $0.281 \mathrm{Ns}$ \\
\hline $\begin{array}{l}\text { Using assistive device } \\
\text { Use cane } \\
\text { Use wall } \\
\text { Support through sons } \\
\text { Don't use }\end{array}$ & $\begin{array}{c}8 \\
8 \\
- \\
14\end{array}$ & $\begin{array}{c}26.7 \\
26.7 \\
- \\
46.7\end{array}$ & $\begin{array}{c}4 \\
12 \\
8 \\
6\end{array}$ & $\begin{array}{l}13.3 \\
40.0 \\
26.7 \\
20.0\end{array}$ & 0.092 & $0.127 \mathrm{Ns}$ \\
\hline
\end{tabular}


Table (4): Mean and standard deviation of Patients' knowledge through pre, post I and post II for both group

\begin{tabular}{|c|c|c|c|c|c|}
\hline \multirow{2}{*}{$\begin{array}{l}\text { Patients' } \\
\text { knowledge }\end{array}$} & & Study & Control & \multirow{2}{*}{ T. test } & \multirow{2}{*}{ P. Value } \\
\hline & & Mean \pm SD & Mean \pm SD & & \\
\hline \multirow{3}{*}{$\begin{array}{l}\text { Knowledge } \\
\text { about concept } \\
\text { of stroke } \\
\end{array}$} & Pre test & $0.9333 \pm 1.63861$ & $0.9333 \pm 1.63861$ & 0.0001 & $1.000 \mathrm{Ns}$ \\
\hline & Post test I & $20.2667 \pm 2.27328$ & $0.9333 \pm 1.63861$ & 37.788 & $0.0001 * *$ \\
\hline & Post test II & $18.0667 \pm 3.05053$ & $0.9333 \pm 1.63861$ & 27.101 & $0.0001 * *$ \\
\hline \multirow{3}{*}{$\begin{array}{l}\text { Knowledge } \\
\text { about activities } \\
\text { of daily living } \\
\text { after stroke } \\
\end{array}$} & Pre test & $0.9333 \pm 1.63861$ & $0.9333 \pm 1.63861$ & 0.0001 & $1.000 \mathrm{Ns}$ \\
\hline & Post test I & $29.333 \pm 2.53708$ & $0.9333 \pm 1.63861$ & 47.384 & $0.0001 * *$ \\
\hline & Post test II & $28.0000 \pm 2.28941$ & $0.9333 \pm 1.63861$ & 47.384 & $0.0001 * *$ \\
\hline \multirow{3}{*}{$\begin{array}{l}\text { Total score of } \\
\text { knowledge }\end{array}$} & Pre test & $1.8667 \pm 2.51524$ & $1.8667 \pm 2.51524$ & 0.0001 & $1.000 \mathrm{Ns}$ \\
\hline & Post test I & $49.6667 \pm 4.46699$ & $1.8667 \pm 2.51524$ & 51.071 & $0.0001 * *$ \\
\hline & Post test II & $46.0667 \pm 5.08502$ & $1.8667 \pm 2.51524$ & 42.674 & $0.0001 * *$ \\
\hline
\end{tabular}

Table (5): Distribution of patient Activities of Daily Living according to Barthel index scale through pre, post I and post II for both group.

\begin{tabular}{|c|c|c|c|c|c|c|c|c|c|c|c|c|c|c|}
\hline \multirow{3}{*}{ Barthel index } & \multicolumn{6}{|c|}{ Control Group } & \multicolumn{6}{|c|}{ Study group } & \multirow{3}{*}{ T test } & \multirow{3}{*}{$P$ value } \\
\hline & \multicolumn{2}{|c|}{ Pre test } & \multicolumn{2}{|c|}{ Post I } & \multicolumn{2}{|c|}{ Post II } & \multicolumn{2}{|c|}{ Pre test } & \multicolumn{2}{|c|}{ Post I } & \multicolumn{2}{|c|}{ Post II } & & \\
\hline & No & $\%$ & No & $\%$ & No & $\%$ & No & $\%$ & No & $\%$ & No & $\%$ & & \\
\hline $\begin{array}{l}\text { Very severely } \\
\text { disabled }\end{array}$ & 6 & 20 & 6 & 20 & 2 & 6.7 & 30 & 100 & 8 & 26.7 & 0 & 0 & 3.221 & $0.0001 * *$ \\
\hline severely disabled & 20 & 66.7 & 20 & 66.7 & 22 & 73.3 & 0 & 0 & 12 & 40 & 8 & 26.7 & 3.521 & $0.0001 * *$ \\
\hline $\begin{array}{l}\text { Moderately } \\
\text { disabled }\end{array}$ & 4 & 13.3 & 4 & 13.3 & 6 & 20 & 0 & 0 & 10 & 33.3 & 14 & 46.7 & 3.751 & $0.0001 * *$ \\
\hline Mildly disabled & - & - & - & - & - & - & - & - & - & - & 8 & 26.7 & - & - \\
\hline $\begin{array}{l}\text { Physical } \\
\text { independent }\end{array}$ & - & - & - & - & - & - & - & - & - & - & - & - & - & - \\
\hline
\end{tabular}

Table (6): Distribution of degree of spasticity according to Ashworth scale through pre, post I, post II in both group.

\begin{tabular}{|c|c|c|c|c|c|c|c|c|c|c|c|c|c|c|}
\hline \multirow{3}{*}{$\begin{array}{c}\text { Ashworth } \\
\text { Scale } \\
\text { (Lower } \\
\text { extremities) }\end{array}$} & \multicolumn{6}{|c|}{ Study group } & \multicolumn{6}{|c|}{ Control group } & \multirow{3}{*}{ T. test } & \multirow{3}{*}{ P. value } \\
\hline & \multicolumn{2}{|c|}{ Pre test } & \multicolumn{2}{|c|}{ Post I test } & \multicolumn{2}{|c|}{ Post- test II } & \multicolumn{2}{|c|}{ Pre test } & \multicolumn{2}{|c|}{ Post I test } & \multicolumn{2}{|c|}{ Post- test II } & & \\
\hline & No & $\%$ & No & $\%$ & No & $\%$ & No & $\%$ & No & $\%$ & No & $\%$ & & \\
\hline \multicolumn{13}{|c|}{ Hamstrings } & & \\
\hline 0 & 10 & 33.3 & 14 & 46.7 & 16 & 53.3 & 2 & 6.7 & 2 & 6.7 & 4 & 13.3 & \multirow{4}{*}{33.359} & \multirow{4}{*}{$0.0001 * *$} \\
\hline 1 & 16 & 53.3 & 10 & 33.3 & 12 & 40 & 14 & 46.7 & 14 & 46.7 & 20 & 66.7 & & \\
\hline 2 & 2 & 6.7 & 4 & 13.3 & 2 & 6.7 & 10 & 33.3 & 10 & 33.3 & 6 & 20 & & \\
\hline 3 & 2 & 6.7 & 2 & 6.7 & 0 & 0 & 4 & 13.3 & 4 & 13.3 & 0 & 0 & & \\
\hline \multicolumn{13}{|c|}{ Quadriceps } & & \\
\hline 0 & 14 & 46.7 & 14 & 46.7 & 16 & 53.3 & 2 & 6.7 & 2 & 6.7 & 4 & 13.3 & \multirow{4}{*}{35.972} & \multirow{4}{*}{$0.0001 * *$} \\
\hline 1 & 10 & 33.3 & 10 & 33.3 & 12 & 40 & 14 & 46.7 & 14 & 46.7 & 22 & 73.4 & & \\
\hline 2 & 4 & 13.3 & 4 & 13.3 & 2 & 6.7 & 10 & 33.3 & 10 & 33.3 & 4 & 13.3 & & \\
\hline 3 & 2 & 6.7 & 2 & 6.7 & 0 & 0 & 4 & 13.3 & 4 & 13.3 & 0 & 0 & & \\
\hline \multicolumn{13}{|c|}{ Gastrocnemius } & \multirow{6}{*}{31.722} & \multirow{6}{*}{$0.0001 * *$} \\
\hline 0 & 14 & 46.7 & 12 & 40 & 14 & 46.7 & 2 & 6.7 & 2 & 6.7 & 4 & 13.3 & & \\
\hline 1 & 10 & 33.3 & 10 & 33.3 & 14 & 46.7 & 14 & 46.7 & 14 & 46.7 & 22 & 73.3 & & \\
\hline 2 & 4 & 13.3 & 6 & 20 & 2 & 6.7 & 10 & 33.3 & 10 & 33.3 & 2 & 6.7 & & \\
\hline 3 & 2 & 6.7 & 2 & 6.7 & 0 & 0 & 2 & 6.7 & 2 & 6.7 & 2 & 6.7 & & \\
\hline 4 & 0 & 0 & 0 & 0 & 0 & 0 & 2 & 6.7 & 2 & 6.7 & 0 & 0 & & \\
\hline
\end{tabular}




\begin{tabular}{|c|c|c|c|c|c|c|c|c|c|c|c|c|c|c|}
\hline \multirow{3}{*}{$\begin{array}{c}\text { Ashworth } \\
\text { Scale } \\
\text { (Lower } \\
\text { extremities) }\end{array}$} & \multicolumn{6}{|c|}{ Study group } & \multicolumn{6}{|c|}{ Control group } & \multirow{3}{*}{ T. test } & \multirow{3}{*}{ P. value } \\
\hline & \multicolumn{2}{|c|}{ Pre test } & \multicolumn{2}{|c|}{ Post I test } & \multicolumn{2}{|c|}{ Post- test II } & \multicolumn{2}{|c|}{ Pre test } & \multicolumn{2}{|c|}{ Post I test } & \multicolumn{2}{|c|}{ Post- test II } & & \\
\hline & No & $\%$ & No & $\%$ & No & $\%$ & No & $\%$ & No & $\%$ & No & $\%$ & & \\
\hline \multicolumn{13}{|c|}{ Soleus } & & \\
\hline 0 & 12 & 40 & 12 & 40 & 12 & 40 & 2 & 6.7 & 2 & 6.7 & 4 & 13.3 & \multirow{5}{*}{24.480} & \multirow{5}{*}{$0.0001 * *$} \\
\hline 1 & 10 & 33.3 & 8 & 26.7 & 16 & 53.3 & 14 & 46.7 & 14 & 46.7 & 22 & 73.3 & & \\
\hline 2 & 6 & 20 & 8 & 26.7 & 2 & 6.7 & 10 & 33.3 & 10 & 33.3 & 2 & 6.7 & & \\
\hline 3 & 2 & 6.7 & 0 & 0 & 0 & 0 & 2 & 6.7 & 2 & 6.7 & 2 & 6.7 & & \\
\hline 4 & 0 & 0 & 2 & 6.7 & 0 & 0 & 2 & 6.7 & 2 & 6.7 & 0 & 0 & & \\
\hline \multicolumn{13}{|c|}{ Hamstrings } & & \\
\hline 0 & 10 & 33.3 & 14 & 46.7 & 16 & 53.3 & 2 & 6.7 & 2 & 6.7 & 4 & 13.3 & \multirow{4}{*}{33.359} & \multirow{4}{*}{$0.0001 * *$} \\
\hline 1 & 16 & 53.3 & 10 & 33.3 & 12 & 40 & 14 & 46.7 & 14 & 46.7 & 20 & 66.7 & & \\
\hline 2 & 2 & 6.7 & 4 & 13.3 & 2 & 6.7 & 10 & 33.3 & 10 & 33.3 & 6 & 20 & & \\
\hline 3 & 2 & 6.7 & 2 & 6.7 & 0 & 0 & 4 & 13.3 & 4 & 13.3 & 0 & 0 & & \\
\hline \multicolumn{13}{|c|}{ Quadriceps } & & \\
\hline 0 & 14 & 46.7 & 14 & 46.7 & 16 & 53.3 & 2 & 6.7 & 2 & 6.7 & 4 & 13.3 & \multirow{4}{*}{35.972} & \multirow{4}{*}{$0.0001 * *$} \\
\hline 1 & 10 & 33.3 & 10 & 33.3 & 12 & 40 & 14 & 46.7 & 14 & 46.7 & 22 & 73.4 & & \\
\hline 2 & 4 & 13.3 & 4 & 13.3 & 2 & 6.7 & 10 & 33.3 & 10 & 33.3 & 4 & 13.3 & & \\
\hline 3 & 2 & 6.7 & 2 & 6.7 & 0 & 0 & 4 & 13.3 & 4 & 13.3 & 0 & 0 & & \\
\hline \multicolumn{13}{|c|}{ Gastrocnemius } & & \\
\hline 0 & 14 & 46.7 & 12 & 40 & 14 & 46.7 & 2 & 6.7 & 2 & 6.7 & 4 & 13.3 & \multirow{5}{*}{31.722} & \multirow{5}{*}{$0.0001 * *$} \\
\hline 1 & 10 & 33.3 & 10 & 33.3 & 14 & 46.7 & 14 & 46.7 & 14 & 46.7 & 22 & 73.3 & & \\
\hline 2 & 4 & 13.3 & 6 & 20 & 2 & 6.7 & 10 & 33.3 & 10 & 33.3 & 2 & 6.7 & & \\
\hline 3 & 2 & 6.7 & 2 & 6.7 & 0 & 0 & 2 & 6.7 & 2 & 6.7 & 2 & 6.7 & & \\
\hline 4 & 0 & 0 & 0 & 0 & 0 & 0 & 2 & 6.7 & 2 & 6.7 & 0 & 0 & & \\
\hline \multicolumn{13}{|c|}{ Soleus } & \multirow{6}{*}{24.480} & \\
\hline 0 & 12 & 40 & 12 & 40 & 12 & 40 & 2 & 6.7 & 2 & 6.7 & 4 & 13.3 & & \multirow{5}{*}{$0.0001 * *$} \\
\hline 1 & 10 & 33.3 & 8 & 26.7 & 16 & 53.3 & 14 & 46.7 & 14 & 46.7 & 22 & 73.3 & & \\
\hline 2 & 6 & 20 & 8 & 26.7 & 2 & 6.7 & 10 & 33.3 & 10 & 33.3 & 2 & 6.7 & & \\
\hline 3 & 2 & 6.7 & 0 & 0 & 0 & 0 & 2 & 6.7 & 2 & 6.7 & 2 & 6.7 & & \\
\hline 4 & 0 & 0 & 2 & 6.7 & 0 & 0 & 2 & 6.7 & 2 & 6.7 & 0 & 0 & & \\
\hline
\end{tabular}

0 - No increase in muscle tone.

1-Slight increase in tone giving a "catch" when affected part is moved in flexion or extension.

2-More marked increase in tone but affected part is easily flexed.

3- Considerable increase in tone; passive movement difficult.

4- Affected part is rigid in flexion or extension.

Fig (1) Total correlation between degree of spasticity according to Ashworth scale and Barthel index in pre \& post $I$ in both groups.

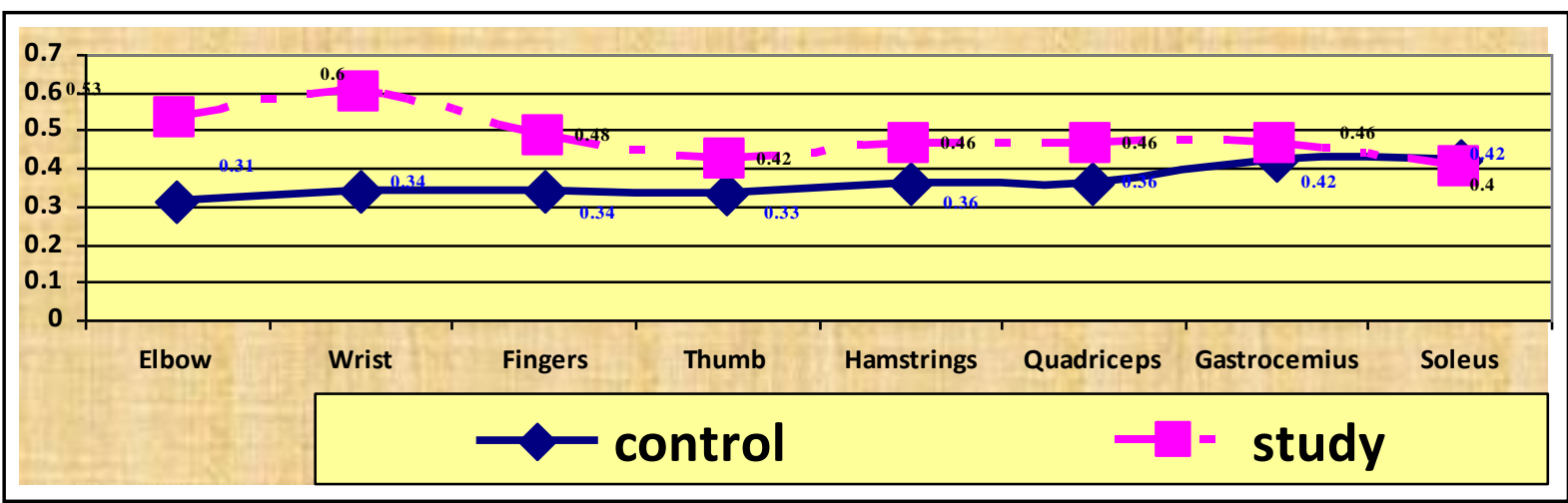


Fig (2) Total correlation between degree of spasticity according to Ashworth scale and Barthel index in post strategies follow up in both group

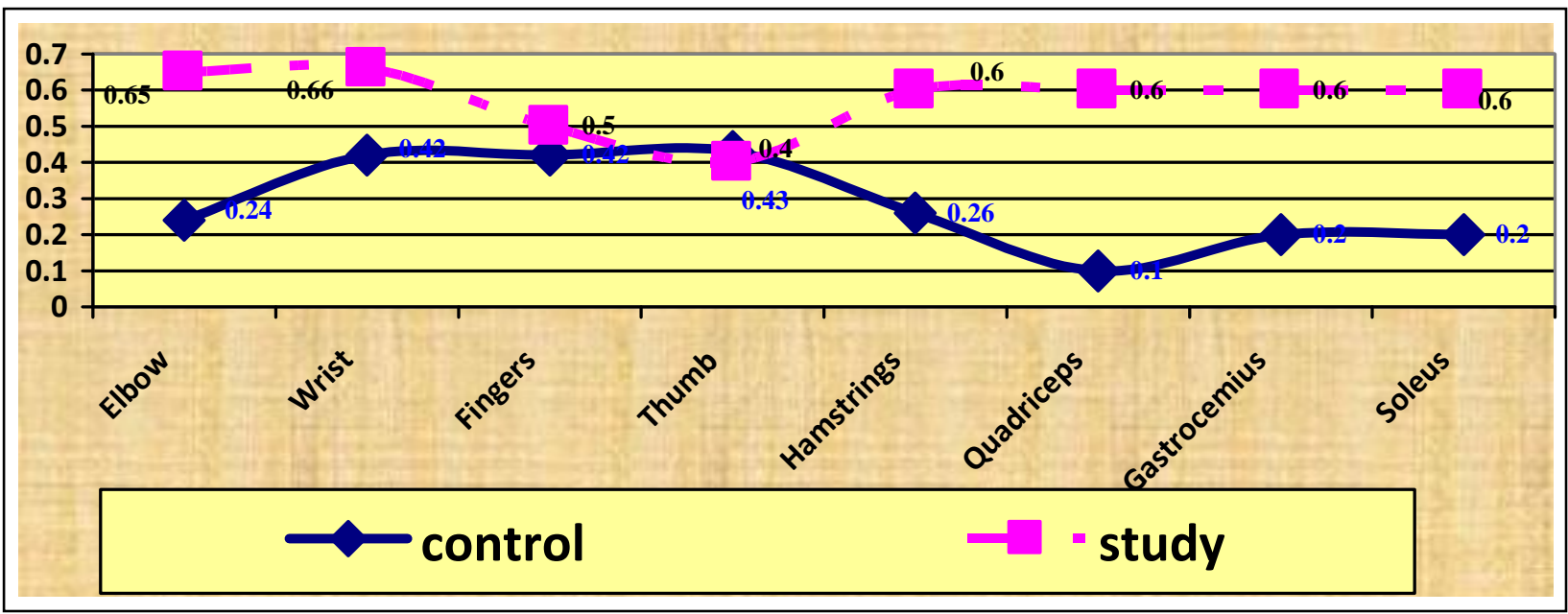

Table (1) : show that; socio-demographic characteristic of the studied group (control and study groups) as regarding to age the majority of both group aged between 51 to 64 years. Regarding to sex it was found that more than half of study group $(53.3 \%)$ were female compared to $(46.7 \%)$ in control group. It was found that, the highest percentages in both groups (study and control) were married, illiterate, house wife and live in urban area $(56.6 \%, 66.6 \%), \quad(73.3 \%, 86.7 \%), \quad(46.7 \%) \quad$ and $(73.3 \%, 60.0 \%)$ respectively.

Table (2) : demonstrates that more than half of study group admitted to hospital in last years (66.7\%) compared to control group $(60.0 \%)$. The highest percentage of control group suffered from pervious cerebral infarction $(46.7 \%)$ which occur in period $2<5$ years $(40.0 \%)$ while the majority of study group not suffered from pervious neurological disease $(73.3 \%)$. More than half of control group suffered from stroke recurrence $(66.7 \%$ ) compared to $26.6 \%$ in study group. As regard to smoking the majority of study group were negative smoker $(46.7 \%)$ and same percentage of control group were cigarette smoker $(46.7 \%)$.

Table (3) : shows that the majority of both groups were having cerebral infarction $(80.0 \%)$ which was developed within 6 month in study \& control groups $(80 \%, 60 \%)$ with affection on dominant hand in study\& control group $(73.3 \%, 60.0 \%)$. less than half from study group use wall as assistive device (40.0\%) and in control group (46.7\%) don't use any assistive device.

Table (4) : show that; increase in total Patients' knowledge among study group after application of strategy $(46.0667 \pm 5.08502) \quad$ compared to $(1.8667 \pm 2.51524)$ in control group with high statistical significant (0.0001).
Table (5) : show that, increase in patients' activities of daily living measured by Barthel index after application of strategy (post test II) than in pre test and post test I in study group than control group this appear through $(26.7 \%)$ improved mildly disabled in study group while the highest degree of patients' activities of daily living performance is moderately disabled (26.7).

Table (6) : show that, the above table that there is decrease in patients' degree of spasticity according to Ashworth scale after application of strategy (post test II) less than in pre and post test I in study group than control group and improvement in lower extremities more than upper extremities and some patient suffered from flaccidity immediately after stroke and convert to increase intensity of muscle tone.

Fig (1) : shows that the correlation between degree of spasticity according to Ashworth scale and activities of daily living according to Barthel index in pre \& post- test I in control group had negative statistical significant correlation in relation to (hamstrings, quadriceps, gastrocnemius and soleus) high of spasticity and low Barthel index based on table of percentage degree of spasticity and Barthel index. But study group in pre \& post- test I had highest strong negative statistical significant correlation (Elbow, wrist, fingers, hamstrings, quadriceps, gastrocnemius) high of spasticity and low Barthel index based on table of percentage degree of spasticity and Barthel index.

Fig (2) : shows that the total correlation between degree of spasticity according to Ashworth scale and Barthel index in control group in post II test had negative statistical significant correlation in relation to (hamstrings, gastrocnemius soleus) high of spasticity and low Barthel index, improvement found in lower extremities only. But in study group had 
highest strong negative statistical significant correlation (Elbow, wrist, fingers, hamstrings, quadriceps, gastrocnemius, soleus) improvement found in upper and lower extremities. Low of spasticity and high Barthel index based on table of percentage degree of spasticity and activities of daily living

\section{Discussion}

The current study revealed that, the high percent of study and control group were female this result supported with Seana et al., (2012) who recently reported that females had more severe strokes, higher short-term mortality, and different management in comparison to males.

The result of the present study revealed that, the majority of patients in both groups aged between 51 to 64 years, the highest percentages in both groups (study and control) were married, illiterate, house wife and live in urban area, this result agree with Zhaoqing et al., (2012) who stated that, the annual incidence of stroke and resulting mortality has increased at an accelerated rate. Furthermore, the incidence of stroke in rural China was higher than that found in urban China and Western countries.

The majority of study group were negative smoker and the highest percentage of control group were cigarette smoking this result agree with Carmona, (2010): with who mention that, smoking nearly doubles the risk for stroke. Smoking causes increased fibrinogen and platelet aggregation. The risk associate with smoking decreases substantially over time after the smoker quiets. After 5 to 10 years of no tobacco used.

The current study revealed a great lack of patient's knowledge as regards to stroke pre the application of strategy either in both study and control group. This finding could be attributed to lack of patient's education about $\&$ how to deal with stroke disease and the highest percentages in both group illiterate, Judi and Valinda, (2012) reported that, use of structured education classes to facilitate the adaptation of stroke survivors who have returned to living in the community

More than 40 percent of stroke patients surveyed reported feeling only some what or not very successful in meeting their rehabilitation goals. Nearly the same percentage of respondents $(38 \%)$ said they lack information on rehabilitation and recovery after stroke. Forty-three percent of the general public surveyed said they wouldn't help, or weren't sure if they would help, someone who had a stroke with daily activities such as food delivery (Russell, 2006).

After implementation of the strategy, patient's knowledge score level were significantly improved in study group but not changed in control group.

Joyce and Jane, (2009) reported that, education is the key to successful treatment of the disease, and the nurse plays a major role as patient educator. Patient and their families need accurate information about the disease and about strategies to minimize its impact. Effective education can alter behavior, empowering patient to make a positive change in their health status.

Public awareness has changed the outlook for stroke. No longer is stroke viewed as fatal or devastating. Education has broadened public knowledge about stroke. Education has taught the public that they have some control over their own health (Osborn et al., 2010).

The result of the present study revealed that, increase in patients' activities of daily living measured by Barthel index after application of strategy (post test II) than in pre test and post test I in study group than control group. Debbie and Janice, (2012) mentioned that, Inpatient rehabilitation may enhance an individual's functional ability after stroke. Stroke patients demonstrated a significant increase in mobility function, and this was accompanied by an increase in daily walking.

Chao et al., (2012) mentioned that, Effects of different intensities of arm rehabilitation training on the functional recovery of hemiplegic upper extremity. An increase in the intensity of arm training might improve the motor function of the arm after stroke.

Stroke is a major cause of long-term disability. About half of the patients surviving for three months after their stroke will be alive five years later, and one third will survive for 10 years. Approximately $60 \%$ of survivors are expected to recover independence with self-care, and $75 \%$ are expected to walk independently. It is estimated that $20 \%$ will require institutional care. The remainder will need assistance either by family. It is noteworthy that psychosocial disabilities (such as difficulties in socialization and vocational functions) are more common than physical disabilities (such as problems with mobility or activities of daily living) (Goldstein, 2006).

Stroke represents one of the most costly and longterm disabling conditions in adulthood worldwide and there is a need to determine the effectiveness of rehabilitation programs in the late phase after stroke for which currently only limited scientific support exists. The general belief has been that treatment of individuals in the late phase of stroke is of no benefit. Today, the concept gives hope for improvements in rehabilitation that go beyond spontaneous recovery of function. The rehabilitation process should encompass all dimensions of a stroke survivor's life, 
and rehabilitation programs that address both the social and physical needs of the patients, preferably individually tailored, are therefore highly desirable. (Lina et al, 2012).

The current study revealed that, decrease in patients' degree of spasticity according to Ashworth scale after application of strategy (post test II) less than in pre test and post test I in study group than control group Yocheved and michal, (2011) reported that, Paretic muscle strength improved after training $(\mathrm{p}<0.05)$ while tone remained consistent $(\mathrm{p}>0.87)$. Gait velocity increased after training $(\mathrm{p}<0.05)$ and at follow-up $(\mathrm{p}<0.05)$. Changes in stair climbing were not significant $(\mathrm{p}>0.37)$, although subjects perceived gains in their physical abilities at follow-up ( $\mathrm{p}<$ $0.01)$.

As regard activities of daily living measured by Barthel index and correlation with knowledge, the result of the present study show that, after application of the strategy, positive statistical significant correlation (high knowledge high Barthel) while knowledge and Barthel index before application of strategy \& post test I and post test II in control group had highest strong positive statistical significant correlation (less knowledge less Barthel index this based on score knowledge of control group).

Stefan et al., (2011) reported that, successful rehabilitation depends on systematic treatment by an interdisciplinary team of experienced specialists, can provide patients with the best functional results. In the area of functional restoration, there has been major progress in our understanding of the physiology of learning, relearning, and training. Further experimental and clinical studies will be needed to expand our knowledge and improve the efficacy of rehabilitation

As regard correlation of spasticity with activities of daily living after application of the strategy in study group, a highest strong negative statistical significant correlation was found in upper and lower extremities (Elbow, wrist, fingers, hamstrings, quadriceps, gastrocnemius, soleus) low of spasticity and high Barthel index based on table of percentage degree of spasticity and barthel while in control group after application of strategy had negative statistical significant correlation in lower extremities (hamstrings, gastrocnemius soleus) low of spasticity and high Barthel index due to progress on lower extremity more than upper and treatment of spasticity progress by use many modalities such as Electrical stimulation to the antagonist muscles or vibrations.

Anthony, (2012) mentioned that, spasticity is a common feature of the upper motor neuron syndrome following stroke. It can have a disabling effect on the stroke survivor through pain and reduced mobility, which may limit the potential success of rehabilitation. Spasticity can also affect quality of life (QoL) and can be both diverse and highly detrimental to daily functioning. Spasticity can result in urinary incontinence; limit sexual intimacy; interfere with walking, sitting, and standing; and generally reduce a person's ability to undertake activities of daily living ADLs.

Stroke is a major cause of chronic impaired arm function and may affect many activities of daily living. At hospital admission after stroke more than two-thirds of all patients have arm paresis, resulting in reduced upper extremity function and six months after stroke the affected arm of approximately half of all patients remains without function. Therefore, to reduce this burden, many patients receive a multidisciplinary rehabilitation approach soon after stroke. Thus, there still exists an urgent need for new inpatient and outpatient rehabilitation and training strategies that match the specific needs of patients and their relatives (Mehrholz et al., 2012).

A recent study examined 103 patients at 6 days, 6 weeks, and 16 weeks post-stroke for change in muscle tone, pain, and paresis. Within 2 weeks of stroke, $24.5 \%$ of patients developed an increase in muscle tone according to the Modified Ashworth Scale. By the first follow-up (median time $=6$ weeks after baseline measurements were recorded), spasticity had emerged in $98 \%$ of patients diagnosed with spasticity throughout the course of the study, and the rate of spasticity at first follow-up had increased only slightly, up to $26.7 \%$. Other predictive factors include a low Barthel Index score (indicating severe disability) at baseline and paresis at any point throughout the study. a history of smoking and all other baseline factors such as sex and age were not associated with severe spasticity. They also had experienced early arm or leg weakness (Wissel et al., 2010).

Despite significant improvements in paretic hand function, no increase in daily use of the paretic or no paretic hand. A disparity between functional recovery and increases in daily use of the upper and lower extremities was found during inpatient stroke rehabilitation (Debbie and Janice, 2012).

Sensory stimulation via TENS may be beneficial to enhance aspects of motor recovery following a stroke, particularly when used in combination with active training. Because of the great variability between studies, particularly in terms of the timing of the intervention after the stroke, the outcome measures used, and the stimulation protocols, insufficient data are available to provide guidelines about strategies and efficacy (Yocheved and michal, 2011). 


\section{Conclusions}

Based on results of the present study the following can be concluded:

Lack patient's knowledge regarding stroke in both study\& control group before application of strategy.

Application of educational strategy for patient with stroke showed an improvement in patients' knowledge in study group. An improvement of patient's knowledge could reflect an improvement on patient's outcomes.

Improvement of patient performing activities of daily (measured by Barthel index) in study group than control group and after than before strategy.

Decrease patient degree of spasticity (measured by Ashworth scale) which may also help in improvement on patient's activities of daily.

\section{Recommendations}

Based on results of the present study the following can be recommended:

For patients'

A continuous educational and training program planned and offered on regular basis to stroke patients in the Rheumatology and Rehabilitation unit. Written, simple booklet about stroke and its management should be provided \& available for patients and their families (relatives)

For further study and research

Replication of the current study on larger probability sample is recommended to achieve generalize ability and wider utilization of the designed program.

Establish an effective training program on nursing intervention of post stroke patients' rehabilitation, should be well organized and equipped with necessary educational facilities for nurses.

\section{References}

1. Abbas M., Fawi1 F., Corea M., Thabit G., and Comi. (2009): Southern EgypPt Stroke Study: Case Fatality Rates in a Hospital-Based Setting General and Internal Medicine Journal, PP 40-46.

2. Anthony B., (2012): Literature Review of the Pathophysiology and Onset of Post Stroke Spasticity, European Journal of Neurology, 19(1): Pp21-27.

3. Asghar D., Sirkka L., Seyed B., Hamid R., and Kristiina H., (2009): Post Stroke Life in Iranian People: Used and Recommended Strategies Iranian, Rehabilitation Journal, 7(9): Pp 17-24.

4. Ashworth B., (1964): Preliminary trial of carisoprodol in multiple sclerosis. Practitioner Pe 540-542.

5. Black J., and Hawks J., (2009): Medical -
Surgical Nursing (Clinical Management for Positive Outcomes). Saunders company, 8th ed., Pp 1844- 1867.

6. Carison K., (2009): AACN Advanced Critical Care Nursing, American Association Critical Care Nurses Saunders 1st ed., Pp 576-316.

7. Chao H., Qiang W., Ping P., Ming-zhu Q., (2012): Effects of intensity of Arm Training on Hemiplegic Upper Extremity Motor Recovery in Stroke Patients: a Randomized Controlled Trial Clinical Rehabilitation, Rehabilitation Journal, 5(16): Pp 122-128.

8. Carmona R., (2010): The Health Consequence of Smoking; a Report of the Surgeon General, Medicine Journal, 12(2): P- 22.

9. Debbie R., and Janice J., (2012): Disparity between Functional Recovery and Daily Use of the Upper and Lower Extremities during Subacute Stroke Rehabilitation, Neurorehabil Neural Repair January, 1(26): Pp 76-84.

10. Debbie R., and Janice J., (2012): Disparity between Functional Recovery and Daily Use of the Upper and Lower Extremities during Subacute Stroke Rehabilitation, Neurorehabil Neural Repair January, 1(26); Pp 76-84.

11. Donnan G., Fisher M., Macleod M., and Davis S., (2008): "Stroke", Neurology Journal, 12(2): Pp 1612-23.

12. Edlin G., and golanty E., (2010)|: health and wellness Jone and Bartlett publishers 10th ed., p.330.

13. Goldstein LB. (2006): Primary Prevention of Ischemic Stroke: a Guideline from the American Heart Association Stroke Council, American Stroke Association, (37): P.1583.

14. Haley W., Roth D., Howard G., (2010): Caregiving Strain and Estimated Risk for Stroke and Coronary Heart Disease among Spouse Caregiver Differential Effects by Race and Sex, Stroke Journal, (41): P. 331.

15. Joyce B., and Jane H., (2009): Medical Surgical Nursing, Library of Congress and Mosby Company 8th ed., p.474.

16. Judi J., and Valinda P., (2012): The Effects of a Structured Education Course on Stroke Survivors Living in the Community, Rehabilitation Nursing Journal, 2(25): Pp 59-65.

17. Kluwer W., (2009): Evidence Based Nursing Guide to Disease Management Lippincott Williams and Wilkins, 1st ed., PP.143-156.

18. Lewis S., Heitkemper M., and Dirksen S., and Bucher L., (2011): Medical Surgical Nursing. Assesment and Management of Clinical problems Mosby, 10th ed., Pr. 788-1525

19. Lina B., Asa L., Christian B., and Michael N., 
(2012): The Effects of a Rhythm and MusicBased Therapy Program and Therapeutic Riding in Late Recovery Phase following Stroke: a Study Protocol for a Three-armed Randomized Controlled Trial, BMC Neurology journals, 12(141): Pp 1471- 2377.

20. Mahoney F., and Barthel D., (1965): Functional Evaluation: the Barthel Index, Maryland State Med Journal, (14): Pp 56-61.

21. Mehrholz J., Hädrich A., Platz T., Kugler J., and Pohl M., (2012): Electromechanical and robot-assisted arm training for improving generic activities of daily living, arm function, and arm muscle strength after stroke (Review), PubMed Journal, 27: Pp 1-52

22. Osborn K., Wraa C., and Waston A., (2010): Medical Surgical Nursing (preparation for practice) pearson 1st ed., P. 757-761.

23. Rodgers C., and Kowalski M., (2008): Text Book of Basic Nursing Lippincott company 9th ed., PP.1241-1245.

24. Russell. (2006): Lack of Adequate Post-Stroke Care Unveiled New Survey Finds the "Forgotten Stroke Survivors" Are Not Receiving Enough Information about Rehabilitation Options, National Stroke Association Journal, 2(4): PP 12-35.

25. Sole M., Klein D., and Moseley M., (2009): Introduction to Critical Care Nursing. Saunders Company, 5th ed., PP. 384 410-420.

26. Seana L., Pham L., Kara M., Leigh B., and Velandai S., (2012): Sex Differences in LongTerm Outcomes After Stroke Functional Outcomes, Handicap, and Quality of Life, American Heart Stroke Association Journal, (8): Pp 1982-1987.

27. Stefan K., Stefan H., and Peter O., (2011): Rehabilitation after Stroke, Neurologische Rehabilitation Journal, 36(108): Pp 600-606.

28. Wissel J., Schelosky L., Scott J., Christe W., Faiss J., and Mueller J., (2010): Early development of spasticity following stroke: a prospective, observational tria, Neurology Journal, (257): Pp 1067-1072.

29. Yocheved L., and Michal E., (2011): Does Sensory Transcutaneous Electrical Stimulation Enhance Motor Recovery Following a Stroke? A Systematic Review. Neuro rehabilitation Journal, 9(25): Pp 799-809

30. Zhaoqing S., Liqiang Z., Robert D., Xingang Z., Jue L., Dayi H., and Yingxian S., (2013):

31. An Epidemiological Survey of Stroke among Rural Chinese Adults Results from the Liaoning Province, International Journal of Stroke, 7(44): P. 1500-1504 